\title{
Improving safety of sedation for transoesophageal echocardiography
}

\author{
Amour Patel, Dev R Katarey
}

\begin{abstract}
This project aimed to develop solutions for optimal, safe sedation, in transoesophageal echocardiagraphy (TOE). Prescription and dosage errors arise from physicians failing to approach experienced operators for assistance, and lack of familiarity with Trust TOE guidelines. We conducted a retrospective analysis of 319 patients who were referred for TOE over a nine-month period. Data was obtained from the hospital medical records and database. Midazolam doses exceeding Trust guidelines were being administered, posing unnecessary risks for under 18 and over 60 year-olds especially. This quality improvement project intervened by: 1 . Adding a paediatric section with sedation dosage advice to Trust guidelines; 2. Increasing awareness of the need for lower sedation doses in over 60 year-olds, by updating the pre-procedure checklist with an age and maximum recommended midazolam dose section. These changes were accepted following presentation of the audit at the monthly Clinical Governance Meeting, and early indications reported increased confidence in midazolam dosing and awareness of the risks of not following the guidelines. Operator confidence in these changes requires re-audit, in order to confirm the findings, and continue improvement to the Trust TOE guidelines and patient safety.
\end{abstract}

\section{Problem}

Optimal, safe prescription and dosage of sedation is an integral part of auditing transoesophageal echocardiagraphy (TOE) service policy. It needs to be effective and well-timed for day cases and inpatients to be discharged safely and efficiently, and remain complication-free during and after the procedure (1). In line with Trust guidelines, patients undergoing TOE are administered an initial dose of intravenous (IV) sedation with midazolam, followed by titration if necessary in small steps up to the maximum recommended dose. A minority of cases experience side-effects (e.g. weakness, fatigue, drowsiness) for the rest of the day, which should resolve thereafter. However, doses exceeding Trust guidelines are being administered, posing unnecessary risks for under 18 and over 60 year-olds in particular.

\section{Background}

We monitored a patient's journey on the day of the procedure, to familiarise ourselves with TOE service policy and identify areas for improvement in the process. Patient details were obtained and checked with the patient, and consent was gained. Trust policy advised that the pre-procedure checklist be completed; however, this was not always complied with. Particularly important points in the checklist, regarding sedation, were that the patient was nil by mouth, an IV cannula had been inserted, an allergy band was in situ, a referral letter was present, and the operator was made aware of any relevant past medical history (eg, diabetes, swallowing difficulties, gastrointestinal bleeding) and current medications. There were one to two patient lists per week, performed by one of six primary operators (one physiologist, five physicians). The medication administration record was documented immediately prior to the procedure to minimise prescribing errors. However, despite the clear Trust guidelines and directions for recording sedation on the checklist, the advice was not always adhered to, especially for over 60 year-olds, debilitated, and chronically ill patients. Trust guidelines were lacking for under 18 year-olds, who were incorrectly categorised as under 60 year-old adults, contradicting the dosage advice provided by the BNF for Children (2). The primary operator recorded baseline, peri- and postprocedure observations and their summary of findings.

Subsequently, all necessary discharge documents were completed and the patient discharged.

\section{Baseline Measurement}

We conducted a retrospective analysis of the 319 patients referred for TOE between September 12012 and May 31 2013. Data was obtained from the hospital medical records and database, and compared with the previous TOE audit, dating January 12011 to August 31 2012. The age and gender distributions remained similar: age range was $17-93$ years, median age was 64 years; $62 \%$ of patients were male. From the referral documentation, the commonest indications for TOE were pre-pulmonary vein ablation (29.7\%), valve assessment (28.4\%) and endocarditis (22.7\%).

\section{Design}

The key to this project was developing long-term solutions to the issues with optimal, safe sedation. We demonstrated $88 \%$ of patients undergoing TOEs are given an IV midazolam $1 \mathrm{mg}$ in $1 \mathrm{ml}$ injection. $5 \%$ were electively given general anaesthetic, whilst $4 \%$ received no sedation at all. Therefore, in line with hospital guidelines midazolam was the sedation agent of choice. Its antagonist, flumazenil, was not administered in any of the 319 cases even though doses exceeding Trust guidelines were being administered, especially in the under 18 and over 60 year-old age groups. After discussions with the cardiac department (including in the monthly Clinical Governance Meeting), changes to practice were proposed. Firstly, paediatric sedation guidelines should be added to the Trust TOE policy, as unlike adults the recommended dose varies according to bodyweight. Secondly, the pre-procedure 
checklist should be updated to include the patient's age and recommended midazolam dose, increasing awareness of requiring lower doses in over 60 year-olds. These solutions would tackle the significant problem of safely dosing children and the elderly.

\section{Strategy}

Our audit demonstrated that the proportion of physician-led TOEs increased by $15 \%$ from the previous audit. This was explained by the loss of the physiologist from the department in December 2012, who had been the leading primary operator, performing over 200 procedures the previous year. Physicians are facing increased demands on their time to perform TOEs. One of the Specialist Registrars was now the leading primary operator in the absence of a physiologist. This pressure on physicians less familiar with Trust guidelines on sedation, coupled with the loss of an experienced physiologist specifically trained in the use of the guidelines, seemed to be reflected by increased deviation from the recommended doses after January 2013. It is becoming clear that the change of personnel operating TOEs is impacting on amount of midazolam administered. The number of TOEs increased from 34 per month to 35 per month (representing a $3 \%$ increase in workload) between the start and end of our audit, despite the loss of a physiologist who conducted a heavy TOE workload and without the addition of new staff. Thus solutions are required to ensure optimal, safe sedation doses are known and adhered to. A long-term solution is for the department to recruit a new specialist physiologist to lead TOE operation. TOE performance data from before and after the loss of the previous physiologist could be looked at, from which the case for a specialist physiologist could be developed, presented, and agreed to by the lead cardiology consultant. Whether the Trust believes the venture is financially feasible at present remains to be seen.

\section{Results}

After serial auditing, all data was scrutinised on a case-by-case basis to identify patients affected by a mismatch between midazolam doses given by operators and those recommended by Trust guidelines. We highlighted the four under 18 year-old cases, as the advice from the BNF for Children had not been adhered to (2). Consultants argued that the recommended doses simply served as guidance to operators unfamiliar with sedation, and that these doses were significantly inadequate. Therefore, in the absence of the patient developing complications, it was a consultant's decision to increase the dose. One 17 year-old, weighing $46 \mathrm{~kg}$, received $10 \mathrm{mg}$ IV midazolam before her TOE, as sedation proved particularly difficult. This calculated to over four times the recommended dose according to her bodyweight, and exceeded the maximum total dose by $2.5 \mathrm{mg}$ for any child her age. The BNF for Children states, "child $12-18$ years: initially $25-50 \mathrm{mcg} / \mathrm{kg}$, increased if necessary in small steps (maximum total dose $7.5 \mathrm{mg}$ )" (2). However, 6-12 year-olds can receive this increment to a maximum total dose of $10 \mathrm{mg}$. Therefore, to minimise sedation errors we added a paediatric component to Trust TOE guidelines. This was met with a reported increase in confidence in prescribing midazolam, when comparing before and after the amendment to guidelines $(20 \%$ versus $80 \%, n=10, p<0.05)$.

Our audit demonstrated that the median midazolam dose had increased from last year, from $3.7 \mathrm{mg}$ to $3.9 \mathrm{mg}$. Out of the 274 cases given midazolam, there were 32 instances of $61-69$ year-olds being given too much according to current hospital policy (39.5\%), and 23 instances of $70-93$ year-olds being given too much (25\%); only $6.9 \%$ of under 60 year-olds were administered too much. It is clear that those over 60 are at much higher risk of midazolam overdosing than those under 60 years of age $(p<0.05)$. The fact that guidelines state the maximum total dose should be $7.5 \mathrm{mg}$ for under 60 year-olds but $3.5 \mathrm{mg}$ for over 60 year-olds seemed a critical factor, emphasising that accessibility, familiarity and adherence to Trust guidelines requires improvement. Two over 60 year-old cases experienced documented side-effects of midazolam during the procedure. One 85 year-old required a rapid IV infusion of $200 \mathrm{ml}$ normal saline to correct hypotension; one 63 year-old experienced a brief episode of supraventricular tachycardia. Studies support updating the pre-procedure checklist, in order to tackle failing aspects and enable safer dosing (3). We increased confidence with correct midazolam dosing, when comparing before and after guideline amendment, by introducing new sections for the patient's age and subsequently their recommended midazolam dose $(30 \%$ versus $90 \%, n=10, p<0.05$ ).

\section{Lessons and Limitations}

This quality improvement project increased our awareness of the patience required to exact change to practice, even with a plausible concept in mind. Data analysis to identify areas for improvement was only a fraction of the challenge. Extensive discussions with all members of the cardiac department (including consultants, pharmacists, nurses and Trust management) proved difficult to arrange. Presenting the audit at the monthly Clinical Governance Meeting was an ideal environment for a discussion forum between all parties for the proposed changes. It enabled documented change to be made more efficiently. Given our tenacity, the subsequent benefit to TOE patient safety was all the more rewarding.

\section{Conclusion}

The initial challenge was to correct unsafe dosing of under 18 and over 60 year-olds with midazolam. It became clear that although Trust TOE policy was readily accessible via the intranet, dosing guidance for children was missing and a lack of attention was drawn to the differences between doses for under and over 60 yearolds. The loss of the experienced physiologist, who was more familiar with Trust TOE policy due to legal prescription limitations, than physicians who have additional time and multitasking pressures, may lead to midazolam dosing inconsistencies. Although the department includes a cardiac pharmacist who helped design the guidelines and could clarify issues with midazolam dosing, they may not be available immediately at the time of TOE. Following extensive analysis and discussion with the department, our solutions for the improvement of hospital TOE policy and patient safety were to: 
1. Add a paediatric section with sedation dosage advice to Trust guidelines.

2. Increase awareness of requiring lower sedation doses in over 60 year-olds, by updating the pre-procedure checklist with an age and maximum recommended midazolam dose section (these changes were accepted).

3. Consideration for the return of a specialist physiologist as lead TOE operator, given the $3 \%$ increase in TOE workload between the start and end of our audit following the loss of a physiologist who contributed heavily to the TOE workload burden and without the addition of new staff.

Re-audit is necessary to determine efficacy of interventions in the various areas studied.

\section{References}

1. Flachskampf FA, Badano L, Daniel WG, Feneck RO, Fox KF, Fraser AG, Pasquet A, Pepi M, Perez de Isla $L$,

Zamorano JL; European Association of Echocardiography; Echo Committee of the European Association of Cardiothoracic Anaesthesiologists, Roelandt JR, Piérard L. Recommendations for transoesophageal echocardiography: update 2010. Eur J Echocardiogr. 2010 Aug;11(7):557-76

2. Paediatric Formulary Committee (2013). British national formulary for children 2013. London: British Medical Association, the Royal Pharmaceutical Society of Great Britain, the Royal College of Paediatrics and Child Health, and the Neonatal and Paediatric Pharmacists Group

3. Gordon BM, Lam TS, Bahjri K, Hashmi A, Kuhn MA. Utility of preprocedure checklists in the congenital cardiac catheterization laboratory. Congenit Heart Dis. 2013 Jun 11. doi: $10.1111 /$ chd.12107. [Epub ahead of print]

\section{Declaration of interests}

Nothing to declare 
Erratum: Improving safety of sedation for transoesophageal echocardiography

Patel A, Katarey DR. Improving safety of sedation for transoesophageal echocardiography BMJ Quality Improvement Reports 2013;2:u202226.w1113. doi:10.1136/bmjquality. u202226.w1113. Since this report was first published online Dev R Katarey has been made a joint first author.

Open Access This is an open-access article distributed under the terms of the Creative Commons Attribution Non-commercial License, which permits use, distribution, and reproduction in any medium, provided the original work is properly cited, the use is non commercial and is otherwise in compliance with the license. See:

- http://creativecommons.org/licenses/by-nc/2.0/

- http://creativecommons.org/licenses/by-nc/2.0/legalcode

BMJ Quality Improvement Reports 2016;5:u202226.w1113corr1. doi:10.1136/bmjquality.u202226.w1113corr1 